\title{
The Dynamic Constants of Unidirectional Fibrous Composites at the Glass Transition Region as Obtained from the Interphase Concept
}

E. Sideridis: School of Applied Mathematics and Physical Sciences NTU Athens, Section of Mechanics, 5 Heroes of Polytechnion Avenue, GR - 15773 Athens, Greece.

Telephone: +302107721224

Fax: +302107721302

Email: siderem@central.ntua.gr

J. Venetis: School of Applied Mathematics and Physical Sciences NTU Athens

Email: johnvenetis4@gmail.com

\begin{abstract}
Dynamic mechanical analysis (DMA) is a versatile technique that complements the information given by the more traditional thermal analysis techniques such as differential scanning calorimetry (DSC), thermogravimetric analysis (TGA), and thermal mechanical analysis (TMA). Dynamic constants such as storage modulus, loss modulus, and loss factor are temperature dependent and provide information about interfacial bonding between reinforced fibre and polymer matrix of composite material. To study the above mentioned properties at the glass transition region, for unidirectional fibrous composites reinforced with continuous fibers a reliable model was applied. In particular, the composite material was considered as composed of three phases with the intermediate phase between matrix and fibres, the interphase, to have variable properties depending on those of main phases and the mode of preparation of the overall material. The glass transition temperature is defined as the point at which the specific volume versus temperature curve changes abruptly slope marking the region between rubbery polymer and glassy polymer nature. Hence, the behaviour of unidirectional fibrous composites was investigated at this region. Examination of the glass transition temperature, which constitutes an upper limit for the structurally important glassy region through the loss factor, was performed by its consideration as a combination of glass transition temperature of matrix and interphase.
\end{abstract}

\section{Keywords}

unidirectional fibrous composites, interphase, storage/loss modulus, loss factor, frequency, glass transition.

\section{Introduction}

It is well known that in lightweight structures, large specific stiffness and strength are aiming properties for a material. By combining fibers with an appropriate polymer and by controlling the production procedure it is possible to make composites featuring large specific properties. Moreover, the constituents are usually cheap and easily processed e.g., by injection molding. Hence, fibrous composites have more and more industrial applications where high performance per weight at a reasonable price is required. One of the most useful forms of composites for the construction of high - performance structural elements, is the type of panels made from aligned fibers containing polymerized matrix. Evidently, their properties depend on the elastic properties and volume fractions of the constituent materials, the fiber length or aspect ratio, the degree of the alignment, the adhesion between fibers and matrix and finally they are affected by the fabrication techniques [1].

The in-plane mechanical properties of a polymer fibre composite are highly dependent on the fibre orientation as the highest stiffness and strength can be obtained when the composite material is loaded in 
the fibre direction. It has been ascertained that the greater the particle fineness of the filler the greater its influence on the kinetics of polymerization. It has also been pointed out that the incorporation of filler affects the polymer concentration at which a continuous three - dimensional system having improved properties is formed. A result of these phenomena is a decrement of the number of possible conformations of molecules present in the boundary layer and the formation of a denser material in the boundary layers than in the bulk. From the above discussion it is apparent that around an inclusion embedded in a matrix a rather complex situation exists consisting of areas of imperfect bonding, where mechanical stresses are abundantly developed as an effect of shrinkage and high stress gradients or even stress singularities, due to the geometry of the inclusion, are evident and after all voids, microcracks and other similar discontinuities may be created. In this case the composite may be considered as consisting of three phases, i.e. the two main phases and a third one, the interphase, which may also arise during thermal treatment of the material because of component interaction [See Fig. 1].

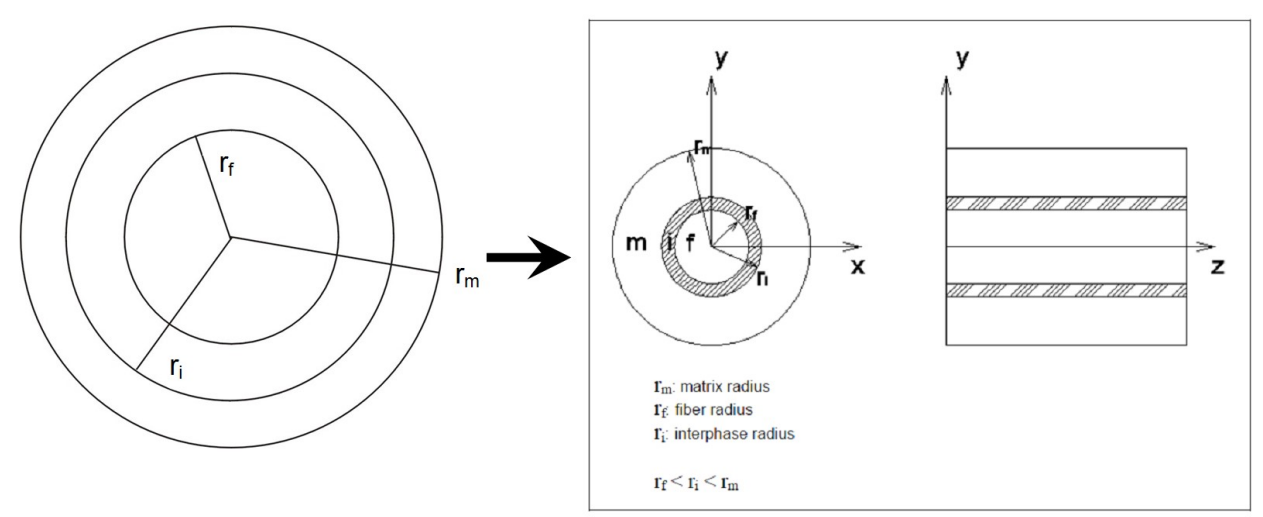

Fig. 1 Cross - sectional area and the three phase cylinder model

The existence of an interphase layer has been proved by infra - red spectroscopy, ESP, NMR, electron microscopy and other experimental methods [2]. Moreover, it has been also proved that the thickness of this layer depends on the polymer cohesion energy, free surface energy of the solid and on the flexibility of the polymer chains [See Fig. 2].

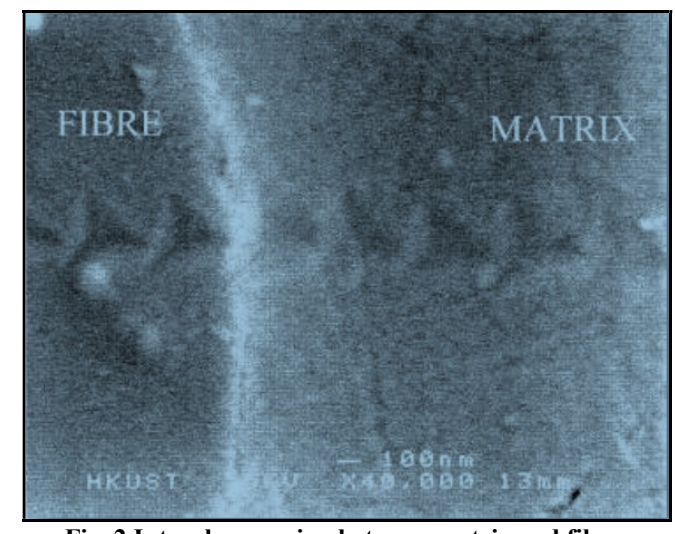

Fig. 2 Interphase region between matrix and fibers

In a model developed by Theocaris et al [3] this intermediate phase has been considered initially as being a homogeneous and isotropic material. In a better approximation [4] a more complex model has been introduced, according to which the fiber was surrounded by a series of successive cylinders, each one of them having a different elastic modulus in a step-function variation with the polar radius.

Moreover, mathematical analyses of inhomogeneous interphases started, probably, with the work of Kanaun and Kudryavtseva [5] on the effective elasticity of a medium with inclusions surrounded by radially inhomogeneous interphase zones, where a rigorous analysis was carried out. Ideas of this work have appeared again in a number of later works. For instance, the basic idea of replacing inhomogeneous inclusions by equivalent homogeneous ones has been utilized in the majority of 
works on the topic; the idea of approximating radially variable properties by multiple layers (piecewise constant variation of properties) was proposed by Garboczi and Bentz [6]. Moreover, several explicit solutions for two specific forms of radial variation of properties, i.e. the linear and the power law ones, have been derived. Specifically, in Ref. [7] an application of power law variation, took place. As far as an arbitrary law of radial variation in properties is concerned, the methodology was proposed by Shen and Li [8], whereby the thickness of the interface is increased in an incremental, "differential" manner, with homogenization at each step. Meanwhile, $\mathrm{Fu}$ et al [9] applied two approaches i.e. the rule of mixtures along with laminate analogy approach in order to describe the elastic modulus of a ternary mixture of particle - fiber - polymer.

On the other hand, the dynamic Tg is defined as the temperature where (i) the middle point of E' vs. temperature curve or (ii) the region where $E^{\prime}$ increases with increasing frequency at constant temperature or (iii) maximum of the tan $\delta$ occurs or (iv) maximum of the $E^{\prime \prime}$ occurs [10]. Thus glass transition temperature is the temperature range where a thermosetting polymer changes from a "glassy", rigid or hard state to a more compliant, pliable or "rubbery" state. It is quite different from melting temperature (Tm), as at melting point the materials begin to melts while at (Tg) the materials get softer.

If the polymer has both crystalline and amorphous form, melting temperature of crystalline form is always higher than the Tg. Moreover, it is the temperature above which the polymer is in the rubbery stage and below to this temperature the polymer is in the glassy or in brittle stage. The glass transition is a transition which happens to amorphous polymers at the glass transition temerature denoted by Tg. Some polymers are used above their glass transition temperatures, and some are used below. Rubber elastomers like polyisobutylene and polyisoprene are used in the rubbery state, i.e. above their $\mathrm{Tg}$ where they are flexible and soft. Hard plastics like poly (methyl methacrylate) and polystyrene are used in glassy state below their glass transition temperatures as their $\mathrm{Tg}$ are around $100 \mathrm{C}^{0}$ well above room temperature. Higher the Tg, greater the cross-linked density which then leads to higher polymer modulus value of the system. The effects of crosslinking on the various regions of the DMA curve are visible in rubbery and glass transition region. However, in the glassy region, both the loss and storage moduli are independent of the degree of cross linking. Thus, highly cross-linked thermoset polymer has much larger loss and storage moduli indicating the tighter network structure and higher stiffness while the polymer of lightly cross linked shows considerable smaller storage and loss modulus.

The glass transition is sensitive to frequency, increasing as frequency increases, but decreasing with increasing amount of plasticizer [10].

All techniques available for the study of the glass transition involve time-dependence in some form or another, since the static tests are time dependent through the rate of temperature change, while dynamic tests use a time dependent- force field and so they have two superposed time dependencies.

A number of loss peaks characterizes the dynamic mechanical spectrum of amine-cured epoxies, which are related to glass transitions and they are associated with cooperative rotational motions, normally involving 20-50 atoms along the main chain. The problem of relating dynamic data to thermomechanical scanning data is complex, as does the very practical problem of deriving from dynamic data a transition temperature suitable for material specifications. The question of how low a frequency is necessary to correlate with dilatomery has been an important point.

In composites, the added reinforcing agent often dilutes the magnitude of changes in physical properties, thus reducing the ability to measure them by classical techniques. Because the elastic properties defined by moduli and the damping properties, measured by dynamic mechanical analysis (D.M.A.), change much more dramatically than heat capacity or thermal expansion, D.M.A. is more sensitive to secondary transitions. There is a considerable disagreement as to the effect of the filler on the glass - transition temperature. While Landel and Smith [11] reported a linear relationship between increased filler-content and increased $T_{g}$ for two binders and two propellant systems, Schwarzl [12] found no such relationship for his urethane-chloride system. His data showed that $T_{g}$ is independent not only of filler content, but also of filler size for variations in particle-size from 1 to $500 \mu$. Turner [13], in his studies on glass - fiber reinforced methacrylate, showed that the glass-rubber transition is not affected appreciably by the glass fibers, even at volume contents as high as 45\%. Kumins and Roteman [14] indicated that $T_{g}$ values of $\mathrm{TiO}_{2}$-filled polyvinyl-acetate were either higher or lower, than that of the original unfilled polymer, depending on the volume fraction of the solid. The magnitude of these changes was in some cases as great 
as $49{ }^{\circ} \mathrm{C}$. Theocaris et al [15], investigated the variation of glass transition temperature with direction in unidirectional glass fiber-reinforced composites.

Dynamic mechanical analysis (DMA) has been increasingly used for the study and characterization of the mechanical properties of materials in general, and of composite materials in particular, owing to the dynamic loading situations frequently found where composites are used [16]. Dynamic mechanical analysis is related to the mobility of the polymer chain segments, and it has been widely used in studies on composite materials for the characterization of interphase properties [17,18]. While dynamicalmechanical techniques have been established for homogeneous materials, a more careful approach for anisotropic materials is currently required. The correct measurement of viscoelastic properties of a material is subject to discussion and depends on equipment used. The measurement is greatly affected by such factors as: heating rate, because a high speed may introduce errors due to the low rate of heat transfer; size of the specimen, because big samples may generate large temperature gradients; adhesion of the matrix-fiber interface, because poor adhesion affects the load transfer; alignment of the fibers; homogeneity of the sample; presence of bubbles; etc. Vazquez et al. [19] analyzed the effect of the interphase modification on the viscoelastic properties of composites. The amount of coating was varied and an optimum coating thickness-to-fiber radius ratio of $1.2 \%$ was found for the diepoxy coating. Alvarez et al [20] performed a valuable investigation on Storage modulus, loss modulus, and loss factor and interphase - fiber / matrix evaluation of unidirectional fiber composites of epoxy resin with glass fibers. In a very recent paper, a thorough review work on dynamic mechanical properties of natural fibre reinforced polymer composites was performed by Saba et al in Ref. [21].

Finally, in Ref. [22] the storage and loss modulus for a general category of Chopped Strand Mat (CSM) composite materials, at their glass transition region, were calculated in an analytical manner by considering the concept of boundary interphase between fibers and matrix.

In the present investigation, a three - phase coaxial cylinder model has been taken into consideration, where the intermediate phase between matrix and fibers plays an important role for the evaluation of $T_{g}$.

This phase is assumed to have variable properties depending on the properties of the two main phases together with the mode of preparation of the composite material. In this context, the storage and loss modulus along with loss factor of a general category of uniaxial fibrous composites at the glass transition region, are studied.

\section{Theoretical Analysis}

The law of mixtures, which is an approximate formula for the longitudinal elastic modulus of unidirectional fiber reinforced composites is used by adding a third region, (the interphase), in order to perform this analysis. In this context, the first region is the polymeric matrix, which is considered as viscoelastic, the modulus of which is $\mathrm{E}_{\mathrm{m}}$. The second one consists of the glass fibers with modulus $\mathrm{E}_{\mathrm{f}}$.

The third region, with modulus $E_{i}$, is assumed to encircle the fibers and consists of macromolecules of the polymer with different physicochemical and thermomechanical properties when compared with the macromolecules of the matrix [Figs. 1,2].

Now, let us consider the mixture law which approximates the elastic modulus in the fiber direction for uniaxial composites

$$
E_{L}=E_{\mathrm{f}} U_{\mathrm{f}}+E_{m} U_{m}
$$

where $E_{L}$ denotes the longitudinal modulus of the overall material whereas $U_{\mathrm{f}} ; U_{m}$ the volume fractions of fiber and matrix respectively, with $U_{\mathrm{f}}+U_{m}=1$.

Eqn. (1), by adding the interphase yields

$$
E_{L}=E_{\mathrm{f}} U_{\mathrm{f}}+E_{m} U_{m}+E_{i} U_{i}
$$

where $U_{i}$ is the interphase content and now $U_{\mathrm{f}}+U_{m}+U_{i}=1$ 


\subsection{Analytical estimation of longitudinal dynamic moduli}

When a sinusoidal dynamic load is imposed in a viscoelastic material its response can be described by the complex, frequency - dependent modulus $E^{*}(\omega)$ where $\omega=2 \pi f$ is the angular frequency. The following relationship holds

$E^{*}(\omega)=E^{\prime}(\omega)+\mathrm{i} E^{\prime \prime}(\omega)$

Here, $E^{\prime}(\omega)$ is the storage modulus whereas $E^{\prime \prime}(\omega)$ denotes its loss modulus. The respective expression for the loss factor is

$\tan \delta_{\mathrm{E}}=E^{\prime \prime}(\omega) / E^{\prime}(\omega)$

where $\delta_{\mathrm{E}}$ is the phase lag angle between stress and strain in extension.

This procedure is called the "correspondence principle" and it was developed by Hashin [23]. In this context, effective complex moduli of viscoelastic composites can be estimated on the basis of analytical representations for effective elastic moduli of composites.

Now, by applying the above principle of viscoelasticity to Eqn. (1) we have

$E_{L}^{*}=E_{\mathrm{f}}^{*} U_{\mathrm{f}}+E_{\mathrm{m}}^{*} U_{m} \Rightarrow$

$E_{L}^{\prime}+\mathrm{i} E_{L}^{\prime \prime}=\left(E_{\mathrm{f}}^{\prime}+\mathrm{i} E_{\mathrm{f}}^{\prime \prime}\right) U_{\mathrm{f}}+\left(E_{\mathrm{m}}^{\prime}+\mathrm{i} E_{\mathrm{m}}^{\prime \prime}\right) U_{m}$

where primed symbols denote the longitudinal storage modulus and double primed symbols the longitudinal loss modulus of the composite and its constituents respectively.

By separating real and imaginary parts one obtains

$$
\begin{aligned}
& E_{L}^{\prime}=E_{\mathrm{f}}^{\prime} U_{\mathrm{f}}+E_{\mathrm{m}}^{\prime}\left(1-U_{\mathrm{f}}\right) \\
& E_{L}^{\prime \prime}=E_{\mathrm{f}}^{\prime \prime} U_{\mathrm{f}}+E_{\mathrm{m}}^{\prime \prime}\left(1-U_{\mathrm{f}}\right)
\end{aligned}
$$

In an analogous manner, from Eqn. (2) one infers

$$
\begin{aligned}
& E_{L}^{\prime}=E_{\mathrm{f}}^{\prime} U_{\mathrm{f}}+E_{i}^{\prime} U_{i}+E_{\mathrm{m}}^{\prime}\left(1-U_{\mathrm{f}}-U_{i}\right) \\
& E_{L}^{\prime \prime}=E_{\mathrm{f}}^{\prime \prime} U_{\mathrm{f}}+E_{i}^{\prime \prime} U_{i}+E_{\mathrm{m}}^{\prime \prime}\left(1-U_{\mathrm{f}}-U_{i}\right)
\end{aligned}
$$

where $E_{i}^{\prime} ; E_{i}^{\prime \prime}$ denote the storage and loss moduli of the interphase respectively

Now, in order to assess the influence of the interphase let us compare the above equations.

It can be observed that $E_{L}^{\prime}$ arising from the concept of interphase i.e. Eqn.(7a) is greater than that without interphase i.e. Eqn.(6a)

$E_{\mathrm{f}}^{\prime} U_{\mathrm{f}}+E_{i}^{\prime} U_{i}+E_{\mathrm{m}}^{\prime}\left(1-U_{\mathrm{f}}-U_{i}\right)>E_{\mathrm{f}}^{\prime} U_{\mathrm{f}}+E_{\mathrm{m}}^{\prime}\left(1-U_{\mathrm{f}}\right) \Rightarrow$ 
$E_{i}^{\prime} U_{i}+E_{\mathrm{m}}^{\prime}\left(1-U_{\mathrm{f}}-U_{i}\right)>E_{\mathrm{m}}^{\prime}\left(1-U_{\mathrm{f}}\right) \Rightarrow$

$E_{i}^{\prime} U_{i}+E_{\mathrm{m}}^{\prime}\left(1-U_{\mathrm{f}}\right)-E_{\mathrm{m}}^{\prime} U_{i}>E_{\mathrm{m}}^{\prime}\left(1-U_{\mathrm{f}}\right) \Rightarrow$

$\left(E_{i}^{\prime}-E_{\mathrm{m}}^{\prime}\right) U_{i}>0$

The above inequality is valid since $U_{i}>0$ and $E_{i}^{\prime}>E_{\mathrm{m}}^{\prime}$ because it is considered that $E_{\mathrm{m}}^{\prime}<E_{i}^{\prime}<E_{\mathrm{f}}^{\prime}$ as we shall discuss later.

In a similar manner, by comparing Eqns. (6b), (7b) one may derive the following inequality

$\left(E_{i}^{\prime \prime}-E_{\mathrm{m}}^{\prime \prime}\right) U_{i}>0$

Evidently, the above inequality is justified by the same reasoning.

It is further assumed that the interphase layer consists of material having progressively variable mechanical properties. In order to match the respective properties of the two main phases bounding the interphase, a variable elastic modulus for the interphase may be defined which for reasons of symmetry depends only on the radial distance from the fiber - interphase surface. In other words it is assumed that the interphase layer consists of a series of elementary peels, the mechanical constants of which differ to each other by a quantity (small enough) defined by the law of variation of $E_{i}(r)$.

Thus, the elastic modulus of the composite is now expressed as follows

$E_{L}=E_{\mathrm{f}} U_{\mathrm{f}}+E_{m}\left(1-U_{\mathrm{f}}-U_{i}\right)+\int_{r_{\mathrm{f}}}^{r_{\mathrm{i}}} E_{i}(r) \frac{\left[\pi(r+d r)^{2}-\pi r^{2}\right] h}{\pi r_{m}^{2} h}$

where

$\mathrm{U}_{f}=\frac{r_{f}{ }^{2}}{r_{m}{ }^{2}} ; \mathrm{U}_{i}=\frac{r_{i}^{2}-r_{f}{ }^{2}}{r_{m}{ }^{2}} ; \mathrm{U}_{m}=\frac{r_{m}{ }^{2}-r_{i}{ }^{2}}{r_{m}{ }^{2}}$

and

$r_{\mathrm{f}} ; r_{\mathrm{m}} ; r_{\mathrm{i}}$ are the radii of the fiber, matrix and interphase respectively

and therefore

$E_{L}=E_{\mathrm{f}} U_{\mathrm{f}}+E_{\mathrm{m}}^{\prime}\left(1-U_{\mathrm{f}}-U_{i}\right)+\frac{2}{r_{m}^{2}} \int_{r_{\mathrm{f}}}^{r_{\mathrm{i}}} E_{i}(r) r d r$

Since it was previously admitted that the interphase constitutes a transition zone between fiber and matrix its modulus can be determined by assuming a progressive variation of the interphase properties from those of the fiber to those of the matrix. Generally, the elastic modulus of the intermediate phase can be expressed as an $n$ - degree polynomial with independent variable the radius $r$, as stated by Theocaris [24]. Hence, we can write out,

$E_{i}(r)=A r^{n}+B r^{n-1}+C r^{n-2}+\ldots$

To this end, one may suppose several laws of variation in the modulus of the interphase, such as linear, parabolic, hyperbolic etc. 
The approach that we shall adopt here is the hyperbolic variation of the interphase modulus $E_{i}$ with respect to radius $r$ which is measured perpendicularly to the centroid axis of the model illustrated in Fig.1.

$$
E_{i}(r)=A+\frac{B}{r}
$$

where $A$ and $B$ are quantities which vary with respect to the radii of matrix and fiber.

To estimate these terms, we have to take into account the following boundary conditions:

$$
\begin{aligned}
& r=r_{f} \Rightarrow E_{i}=k E_{\mathrm{f}} \\
& r=r_{i} \Rightarrow E_{i}=E_{m} \\
& \forall r>0: r_{\mathrm{f}} \leq r \leq r_{i}
\end{aligned}
$$

Here we assume that the coefficient of modulus of the interphase, at the interface with the matrix, coincides with the quantity $E_{m}$. Besides, at the interface between interphase and fiber, the quantity $E_{i}$ constitutes actually a portion of $E_{\mathrm{f}}$, which evidently implies that $E_{i}<E_{\mathrm{f}}$.

This influence is indicated by the coefficient $\mathrm{k}$ the values of which belong to the interval $(0,1]$. Hence the following relationship arises

Thus it follows

$$
E_{i}(r)=\frac{E_{m} r_{i}-k E_{f} r_{f}}{r_{i}-r_{f}}+\left[\frac{\left(k E_{f}-E_{m}\right) r_{f} r_{i}\left(r-2 r_{i}\right)}{\left(r_{i}-r_{f}\right)}\right] / r
$$

A mean value for this modulus can be evaluated as

$$
\begin{aligned}
& E_{i}(r)=\frac{1}{V_{i}} \int_{r_{\mathrm{f}}}^{r_{i}} E_{i}(r) d V_{i}=\frac{1}{V_{i}} \int_{r_{\mathrm{f}}}^{r_{i}}(A+B / r) d V_{i} \Leftrightarrow \\
& E_{i}(r)=\frac{2}{r_{i}^{2}-r_{\mathrm{f}}^{2}}\left[\frac{A}{3}\left(r_{i}^{3}-r_{\mathrm{f}}^{3}\right)+\frac{B}{2}\left(r_{i}^{2}-r_{\mathrm{f}}^{2}\right)\right]
\end{aligned}
$$

\section{Determination of the extent of interphase}

Differential scanning calorimetry (DSC) method was used to determine the interphase thickness and volume fraction. If calorimetric measurements are performed in the neighbourhood of the glass transition zone, it is easy to show that jumps of energies appear in this neighborhood. These jumps are very sensitive to the amount of filler added to the matrix polymer and they were used for the evaluation of the boundary layers developed around fibers. Moreover the augmentation of the fiber content increases the proportion of macromolecules which are in contact with fiber surface and are characterized by a reduced mobility. This is equivalent to an increase in interphase volume fraction and leads to the conclusion of Lipatov [25], stating that a relation holds between $\Delta \mathrm{Cp}$ which expresses the sudden change in the heat capacity at the glass transition region, [see Fig. 3], and the interphase thickness $\Delta \mathrm{r}$ and thus interphase volume fraction. 


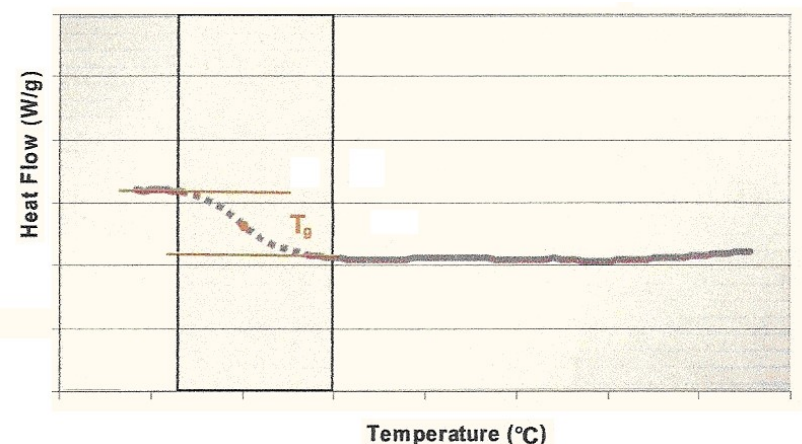

Fig. 3 Typical diagram for the determination of interphase content at the glass transition zone

Hence one writes

$\left(\frac{r_{\mathrm{f}}+\Delta r}{r_{\mathrm{f}}}\right)^{2}-1=\frac{\lambda}{1-U_{\mathrm{f}}}$

where $\Delta r$ is the thickness of the interphase and the parameter $\lambda$ is given by

$$
\lambda=1-\frac{\Delta C_{p}^{\mathrm{f}}}{\Delta C_{p}^{0}}
$$

Here, $\Delta C_{p}^{\mathrm{f}} ; \Delta C_{p}^{0}$ are the sudden changes of the heat capacity for the pure and reinforced matrix respectively.

\section{Materials and Experiments}

The unidirectional glass - fiber composite used in this study consisted of long E - glass fibers (Permaglass XE - B 5/1) embedded in Araldite MY 750 HT 972 epoxy resin based or diglycidyl ether of bisphenol A together with an aromatic amine hardener with $\mathrm{E}_{\mathrm{f}}=70 \mathrm{GPa}$, $\mathrm{E}_{\mathrm{m}}=3.0 \mathrm{GPa}, v_{\mathrm{f}}=0.2, v_{\mathrm{m}}=0.35$.

The glass fibers had a diameter of $12 \times 10^{\wedge}-6 \mathrm{~m}$ and were contained in a volume fraction $\mathrm{U}_{\mathrm{f}}=0.65$. The values of the moduli E'; E" of matrix and composite were measured with a Dynastat and Dynalizer apparatuses. The latter can apply a sinusoidal load of maximum amplitude of $100 \mathrm{~N}$ on a cylindrical specimen mounted between a long, upper rod connected to a load cell and a short lower one coupled to a displacement transducer. By passing a servo controlled current through the coil of transducer the specimen is subjected to a sinusoidal load of given amplitude and frequency. The latter has range from 0.1 to $100 \mathrm{~Hz}$. The results are illustrated in Fig. $(4 \mathrm{a}, \mathrm{b})$

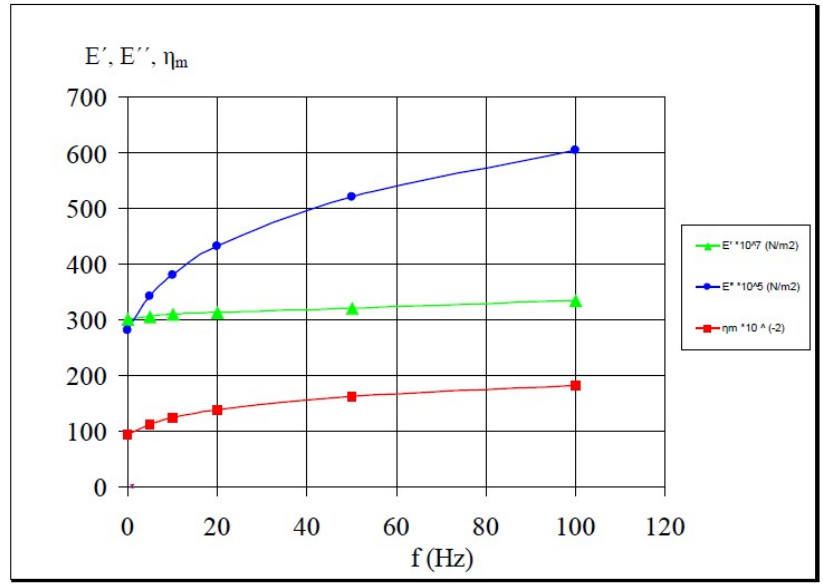

Fig. 4a Dynamic moduli and loss factor of the matrix vs frequency 


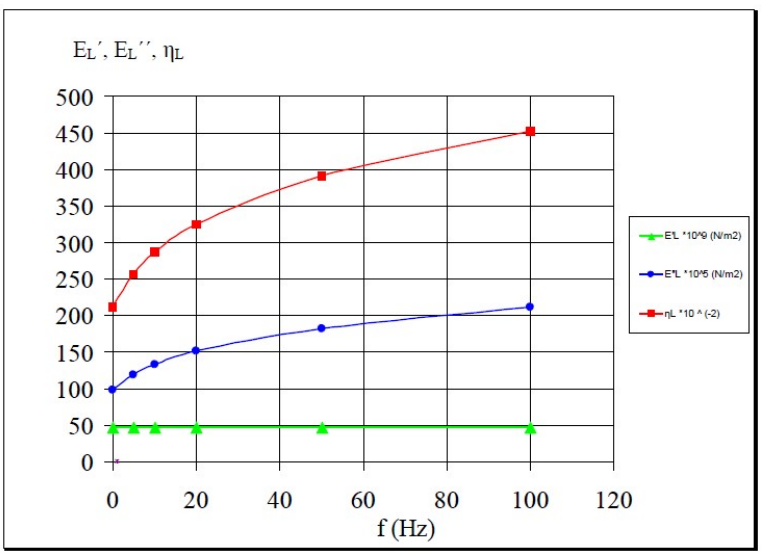

Fig. 4b Longitudinal dynamic moduli and loss factor of the composite vs frequency

In the meanwhile, the specimens with $4 \times 10^{\wedge}-3 \mathrm{~m}$ diameter and thickness between $10 \times 10^{\wedge}-4 \mathrm{~m}$ and 15 $\mathrm{x} 10^{\wedge}-4 \mathrm{~m}$, made of the constituents were tested on a DSC Thermal Analyzer at the zone of $\mathrm{T}_{\mathrm{g}}$ for each mixture, in order to define the thickness $\Delta \mathrm{r}$ and the content of the interphase which was found as $U_{i}=$ 0.052 .

Next, to extend the predictions of the moduli beyond the previously denoted value of filler content, there is a need to assess the interphase thickness and volume fraction for these fiber contents.

In this context, one may assume that this quantity, which vanishes for $U_{\mathrm{f}}=0$, increases, reaching a unique peak value and then decreases tending to zero at $U_{\mathrm{f}} \rightarrow 1$, given that $\mathrm{U}_{\mathrm{f}}=1-\mathrm{U}_{\mathrm{m}}-\mathrm{U}_{\mathrm{i}}$

Of course, in reality the maximum of fiber content approaches to 0.9 .

To perform a better description of interphase content, in terms of $U_{f}$ let us adopt the following continuous function

$U_{i}=C_{1} U_{\mathrm{f}}^{3}+C_{2} U_{\mathrm{f}}^{2}+C_{3} U_{\mathrm{f}}+C_{4}$

To estimate the unknown constants we can choose the experimental value $U_{\mathrm{i}}=0.052$ at $U_{\mathrm{f}}=0.65$ and also apply the boundary conditions $U_{i}=0$ at $U_{f}=0$ and $U_{i}=0$ at $U_{f} \rightarrow 1$, which obviously marked the minimum value of the interphase content and can be determined by the following Neumann boundary conditions: $\frac{\mathrm{dU}_{\mathrm{i}}}{\mathrm{dU}_{\mathrm{f}}}=\left.0\right|_{\mathrm{U}_{\mathrm{f}}=0}$ or $\frac{\mathrm{dU}_{\mathrm{i}}}{\mathrm{dU}_{\mathrm{f}}}=\left.0\right|_{\mathrm{U}_{\mathrm{f}}=1}$

Moreover, whereas the critical value of $\mathrm{U}_{\mathrm{f}}$ which corresponds to the maximum interphase content lies on the interval $[0,1]$.

Hence, one may deduce that the maximum ordinate of this curve, i.e. the maximum $U_{i}$ will be the other extremum (global or local) of this parabola and thus the overall interphase region is known.

Eqn. (19) in accordance with its boundary conditions yields the following values

$C_{1}=-0.3537 ; C_{2}=0.3537 ; C_{3}=0 ; C_{4}=0$

and therefore Eqn. (19) becomes

$U_{i}=-0.3537 U_{f}^{3}+0.3537 U_{f}^{2}$

In Fig. 5, the interphase volume fraction versus filler content is illustrated

\section{Determination of the dynamic moduli}

By applying the correspondence principle of viscoelasticity to the hyperbolic variation and then by separating the real and imaginary parts in each side we have 
$E_{i}^{\prime}(r)=\frac{k E_{\mathrm{f}}^{\prime} r_{\mathrm{f}}\left(r_{i}-r\right)+E_{m}^{\prime} r_{i}\left(r-r_{\mathrm{f}}\right)}{\left(r_{i}-r_{\mathrm{f}}\right) r}$

and

$E^{\prime \prime}{ }_{i}(r)=\frac{k E_{\mathrm{f}}^{\prime \prime}\left(r_{i}-r\right)+E^{\prime \prime}{ }_{m} r_{i}\left(r-r_{\mathrm{f}}\right)}{\left(r_{i}-r_{\mathrm{f}}\right) r}$

Here, since fibers are elastic we can consider that $E_{\mathrm{f}}^{\prime} \cong E_{\mathrm{f}}$ and $E^{\prime \prime}{ }_{\mathrm{f}} \cong 0$

Then, the loss factor of interphase is obtained from the above equations

$\eta_{i}=\tan \delta_{i}=\frac{E^{\prime \prime}{ }_{m}\left(r-r_{\mathrm{f}}\right) r_{i}}{k E_{\mathrm{f}}^{\prime} r_{\mathrm{f}}\left(r_{i}-r\right)+E_{m}^{\prime} r_{i}\left(r-r_{\mathrm{f}}\right)}$

\section{Evaluation of the glass - transition temperature}

As stated previously, the storage and loss moduli depend on the angular frequency $\omega$.

This can be observed from the diagrams in Fig. (4a,b) where, as it was stated, the dynamic moduli with respect to frequency appear. Hence, from Eqns. $(4 a, b)$ we may obtain an expression to find the loss factor of the overall material $\eta_{L}(\omega)$ which mainly depends on the frequency $\omega$ of the above vibrational load. This implies that this factor is a continuous single - valued function of the variable $\omega$. Moreover, this factor is a convenient parameter for the determination of $T_{g}$, since its maximum value corresponds to the center of transition region expressing therefore the amount of energy dissipated as a fraction of the energy stored in the system. At the transition frequency $\omega_{g}$ the loss factor is assumed to have its maximum value [see Fig. 6] hence the following condition holds

$\left.\frac{d \eta(\omega)}{d \omega}\right|_{\omega=\omega_{g}}=0$

In applying the correspondence principle, one can get the following expressions

$E_{m}^{*}=E_{m}^{\prime}(\omega)+\mathrm{i} E_{m}^{\prime \prime}(\omega)=E_{m}^{\prime}\left[1+\mathrm{i} \eta_{\mathrm{m}}(\omega)\right]$

where $\eta_{\mathrm{m}}(\omega)=E_{m}^{\prime \prime}(\omega) / E_{m}^{\prime}(\omega)$

and

$E_{i}^{*}=E_{i}^{\prime}(\omega)+\mathrm{i} E_{i}^{\prime \prime}(\omega)=E_{i}^{\prime}\left[1+\mathrm{i} \eta_{i}(\omega)\right]$

with $\eta_{i}(\omega)=E_{i}^{\prime \prime}(\omega) / E_{i}^{\prime}(\omega)$

Next, let us consider Eqns. (7a,b)

By dividing, we obtain the longitudinal loss factor of the composite

$\eta_{L}(\omega)=\frac{E_{m}^{\prime}(\omega) \cdot \eta_{\mathrm{m}}(\omega) \cdot U_{m}+E_{i}^{\prime}(\omega) \cdot \eta_{i}(\omega) \cdot U_{i}}{E_{\mathrm{f}}^{\prime} U_{\mathrm{f}}+E_{m}^{\prime} U_{m}+E_{i}^{\prime}(\omega) \cdot U_{i}}$

If $\omega_{g, L} ; \omega_{g, m}$ represent the transition frequencies of the composite and the matrix respectively the relative magnitude of these quantities may define any relation between the glass - transition temperatures of the matrix $T_{g, m}$ and of the composite $T_{g, c}$. Indeed, if $\omega_{g, L} \leq \omega_{g, m}$, then $T_{g, c}>T_{g, m}$ and inversely if $\omega_{g, L}>\omega_{g, m}$ it implies that $T_{g, c}<T_{g, m}$. 
The above result is attributed to the fact that the loss factor takes its peak value in the middle of the transition zone where the glass transition is defined.

The relative position of the transition frequencies $\omega_{g, L} ; \omega_{g, m}$ could now be determined by finding the following specific value of the derivative of the term $\eta_{L}(\omega)$, i.e. $\left.\frac{d \eta_{L}(\omega)}{d \omega}\right|_{\omega=\omega_{g, m}}$.

If this quantity is negative it follows that $\omega_{g, L}<\omega_{g, m}$ because in this case the loss factor has already reached its maximum value. Inversely, if it is positive the loss factor will be still in the zone where its values are increasing and therefore it will take its peak value at $\omega_{g, L}$ which is strictly larger than $\omega_{g, m}$.

Thus it follows

$$
\begin{aligned}
& \left.\frac{d \eta_{L}(\omega)}{d \omega}\right|_{\omega=\omega_{g, m}}=\frac{\left(E_{\mathrm{f}}^{\prime} U_{\mathrm{f}}+E_{m}^{\prime} U_{m}+E_{i}^{\prime} \cdot U_{i}\right)\left[\frac{d E_{m}^{\prime}}{d \omega} \eta_{m} U_{m}+E_{m}^{\prime} U_{m} \frac{d \eta_{m}}{d \omega}+\frac{d E_{i}^{\prime}}{d \omega} \eta_{i} U_{i}+E_{i}^{\prime} U_{i} \frac{d \eta_{i}}{d \omega}\right]}{\left(E_{\mathrm{f}}^{\prime} U_{\mathrm{f}}+E_{m}^{\prime} U_{m}+E_{i}^{\prime} \cdot U_{i}\right)^{2}}- \\
& \frac{\left(E_{\mathrm{m}}^{\prime} \eta_{m} U_{m}+E_{i}^{\prime} \eta_{i} U_{i}\right)\left[\frac{d E_{\mathrm{f}}^{\prime}}{d \omega} U_{\mathrm{f}}+\frac{d E_{\mathrm{m}}^{\prime}}{d \omega} U_{m}+\frac{d E_{i}^{\prime}}{d \omega} U_{i}\right]}{\left(E_{\mathrm{f}}^{\prime} U_{\mathrm{f}}+E_{m}^{\prime} U_{m}+E_{i}^{\prime} \cdot U_{i}\right)^{2}}
\end{aligned}
$$

Since the fibers are assumed to behave elastically it implies that

$\frac{d E_{\mathrm{f}}}{d \omega}=\frac{d E_{\mathrm{f}}^{\prime}}{d \omega}=0$

whereas

$\left.\frac{d \eta_{m}(\omega)}{d \omega}\right|_{\omega=\omega_{g, m}}=0$

Thus if one sets

$F=E_{\mathrm{f}}^{\prime} U_{\mathrm{f}}+E_{m}^{\prime}(\omega) \cdot U_{m}+E_{i}^{\prime}(\omega) \cdot U_{i}$

and

$H=\left(\frac{d E_{\mathrm{m}}^{\prime}}{d \omega} E_{i}^{\prime}-\frac{d E_{i}^{\prime}}{d \omega} E_{\mathrm{m}}^{\prime}\right)\left(\eta_{i}-\eta_{m}\right) U_{m} U_{i}$

Eqn. (25) can be equivalently written as

$\left.\frac{d \eta_{L}(\omega)}{d \omega}\right|_{\omega=\omega_{g, m}}=\frac{\left[E_{\mathrm{f}}^{\prime} U_{\mathrm{f}} U_{m} \eta_{m} \frac{d E_{m}^{\prime}}{d \omega}+F \cdot E_{i}^{\prime} U_{i} \frac{d \eta_{i}}{d \omega}+E_{i}^{\prime} \cdot U_{i}+E_{f}^{\prime} U_{f} U_{i} \eta_{i} \frac{d E_{i}^{\prime}}{d \omega}\right]-H}{F^{2}}$

In the above expression, the quantity $\left.\frac{d E_{m}^{\prime}(\omega)}{d \omega}\right|_{\omega=\omega_{g, m}}$ is positive since the storage modulus is always an increasing function of frequency in the glass transition zone as it can be observed in Fig. 4.

It can be said that an analogous reasoning is valid for $\left.\frac{d E_{i}^{\prime}(\omega)}{d \omega}\right|_{\omega=\omega_{g, m}}$ since interphase can be considered as a somewhat altered matrix.

Indeed, from eqn. (20a) we infer 
$\left.\frac{d E_{i}^{\prime}(\omega)}{d \omega}\right|_{\omega=\omega_{g, m}}=\frac{\left[k \frac{d E_{f}^{\prime}}{d \omega}\left(r_{i}-r\right) r_{f}+\frac{d E_{m}^{\prime}}{d \omega}\left(r-r_{f}\right) r_{i}\right]\left(r_{i}-r_{f}\right) r-\left[k E_{f}^{\prime}\left(r_{i}-r\right) r_{f}+E_{m}^{\prime}\left(r-r_{f}\right) r_{i}\right] \frac{d\left(r_{i}-r_{f}\right)}{d \omega}}{\left[\left(r_{i}-r_{f}\right) r\right]^{2}}$

Hence, by considering previous assumptions concerning variations with frequency after the necessary algebraic manipulations one obtains

$$
\frac{d E_{i}^{\prime}(\omega)}{d \omega}=\frac{d E_{\mathrm{m}}^{\prime}(\omega)}{d \omega} \cdot \frac{\left(r-r_{f}\right) r_{i}}{\left(r_{i}-r_{f}\right) r}
$$

One may easily point out that this expression is also strictly positive since the terms $\frac{d E_{\mathrm{m}}^{\prime}}{d \omega}$ and $\frac{\left(r-r_{f}\right) r_{i}}{\left(r_{i}-r_{f}\right) r}$ are also strictly positive because the radii are positive and in the interphase region $r_{f}<r_{i}<r$.

Eqn. (21) and (23a) can be combined to yield

$$
\eta_{i}=\tan \delta_{i}=\frac{\eta_{m} E_{m}^{\prime}\left(r-r_{\mathrm{f}}\right) r_{i}}{k E_{\mathrm{f}}^{\prime} r_{\mathrm{f}}\left(r_{i}-r\right)+E_{m}^{\prime} r_{i}\left(r-r_{\mathrm{f}}\right)}
$$

Now according to the same reasoning let us evaluate the derivative of $\eta_{i}(\omega)$

$\left.\frac{d \eta_{i}(\omega)}{d \omega}\right|_{\omega=\omega_{g, m}}=\frac{\left[k E_{f}^{\prime}\left(r_{i}-r\right) r_{f}+E_{m}^{\prime}\left(r-r_{f}\right) r_{i}\right]\left(\frac{d E_{m}^{\prime}}{d \omega} \eta_{m}+E_{m}^{\prime} \frac{d \eta_{m}}{d \omega}\right)\left(r-r_{f}\right) r_{i}-\left[\eta_{m} E_{m}^{\prime}\left(r-r_{f}\right) r_{i}\right] \cdot\left(k \frac{d E_{f}^{\prime}}{d \omega}\left(r_{i}-r\right) r_{f}+\frac{d E_{m}^{\prime}}{d \omega}\left(r-r_{f}\right) r_{i}\right)}{\left[k E_{f}^{\prime}\left(r_{i}-r\right) r_{f}+E_{m}^{\prime}\left(r-r_{f}\right) r_{i}\right]^{2}}$

Since $\frac{d E_{\mathrm{f}}^{\prime}}{d \omega}=0 ;\left.\frac{d \eta_{\mathrm{m}}}{d \omega}\right|_{\omega=\omega_{g, m}}=0$

and therefore after some algebra we find

$$
\left.\frac{d \eta_{i}(\omega)}{d \omega}\right|_{\omega=\omega_{g, m}}=\frac{k E_{f}^{\prime} \eta_{m} \frac{d E_{m}^{\prime}}{d \omega}\left(r_{i}-r\right)\left(r-r_{f}\right) r_{i} r_{f}}{\left[k E_{f}^{\prime}\left(r_{i}-r\right) r_{f}+E_{m}^{\prime}\left(r-r_{f}\right) r_{i}\right]^{2}}
$$

In the right member of the above expression, one may observe that the denominator of the fraction is positive. Moreover, the quantities $k$ and $E_{f}^{\prime}$ are positive fact that also concerns the term $\eta_{m}$, since $E_{m}^{\prime}$ and $E_{m}^{\prime \prime} \quad$ are positive, and finally $\left.\frac{d E_{\mathrm{m}}^{\prime}}{d \omega}\right|_{\omega=\omega_{g, m}}$ is positive as stated before together with the product $\left(r-r_{f}\right) r_{i} r_{f}\left(r_{i}-r_{f}\right)$, which is positive in interphase zone.

In this context, in eqn. (30) the contribution of the quantity $\frac{d E_{i}^{\prime}}{d \omega}$ is minor when compared to that of $\frac{d E_{\mathrm{m}}^{\prime}}{d \omega}$ since it is multiplied by the interphase content $U_{i}$, the values of which are always smaller that $5 \%$ of the overall material volume, as it can be observed from Fig. 5. 


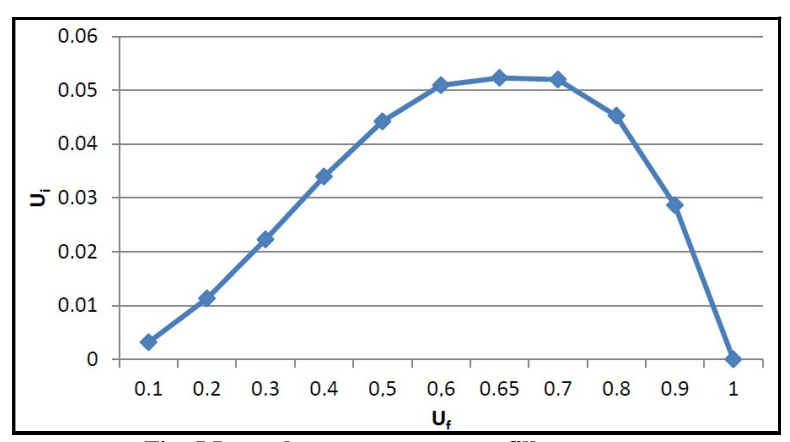

Fig. 5 Interphase content versus filler content

To elucidate the above reasoning, let us consider the following ratio

$$
\frac{\left(d E_{i}^{\prime} / d \omega\right) \eta_{i} U_{i}}{\left(d E_{m}^{\prime} / d \omega\right) \eta_{m} U_{m}}=\frac{\left(d E_{i}^{\prime} / d \omega\right)\left(E_{i}^{\prime \prime} / E_{i}^{\prime}\right) U_{i}}{\left(d E_{i}^{\prime} / d \omega\right)\left(E_{m}^{\prime \prime} / E_{m}^{\prime}\right) U_{m}}
$$

In our previous derivations, we have considered the loss and storage moduli of the matrix and interphase as single - valued continuous functions of the frequency $\omega$. Thus, the following relationships hold

$E_{m}^{\prime}=f_{1, m}(\omega) E_{m}$

$E_{i}^{\prime}=f_{1, i}(\omega) E_{i}$

$E_{m}^{\prime \prime}=f_{2, m}(\omega) E_{m}$

$E_{i}^{\prime \prime}=f_{2, i}(\omega) E_{i}$

Apparently, the previously introduced single - valued continuous functions of the variable $\omega$ arise from the applied rheological model.

Thus, taking into account the Maxwell model, one infers

$$
\begin{aligned}
& f_{1, m}(\omega)=\frac{\omega^{2} \tau_{m}^{2}}{1+\omega^{2} \tau_{m}^{2}} ; f_{2, m}(\omega)=\frac{\omega \tau_{m}}{1+\omega^{2} \tau_{m}^{2}} \\
& f_{1, i}(\omega)=\frac{\omega^{2} \tau_{i}^{2}}{1+\omega^{2} \tau_{i}^{2}} ; f_{2, i}(\omega)=\frac{\omega \tau_{i}}{1+\omega^{2} \tau_{i}^{2}}
\end{aligned}
$$

On the other hand, the consideration of Voigt model yields

$$
\begin{aligned}
& f_{1, m}(\omega)=1 ; f_{2, m}(\omega)=\omega \tau_{m} \\
& f_{1, i}(\omega)=1 ; f_{2, i}(\omega)=\omega \tau_{i}
\end{aligned}
$$

where $\tau_{m}, \tau_{i}$ denote the relaxation time of matrix and interphase respectively.

It can be assumed, without violating the generality, that the relaxation time $\tau_{m}$ of the matrix and that of interphase $\tau_{i}$ are approximately equal, i.e. $\tau_{m} \cong \tau_{i}$

Hence, eqn. (35) becomes

$$
\frac{\left(d E_{i}^{\prime} / d \omega\right) \eta_{i} U_{i}}{\left(d E_{m}^{\prime} / d \omega\right) \eta_{i} U_{i}}=\frac{\left(d E_{i}^{\prime} / d \omega\right)\left(E_{i}^{\prime \prime} / E_{i}^{\prime}\right) U_{i}}{\left(d E_{i}^{\prime} / d \omega\right)\left(E_{m}^{\prime \prime} / E_{m}^{\prime}\right) U_{m}}
$$


Meanwhile, one may pinpoint that the positive quantity $H$ also has a small contribution in in eqn. (30) since it can be modified as

$$
\begin{aligned}
& H=\left.\left(\frac{d E_{\mathrm{m}}^{\prime}}{d \omega} E_{i}^{\prime}-\frac{d E_{i}^{\prime}}{d \omega} E_{\mathrm{m}}^{\prime}\right)\left(\eta_{i}-\eta_{m}\right) U_{m} U_{i}\right|_{\omega=\omega_{g, m}} \Leftrightarrow \\
& H=\left.\left(E_{\mathrm{i}}^{\prime \prime}\left(\omega_{g, m}\right)-E_{\mathrm{m}}^{\prime \prime}\left(\omega_{g, m}\right)\right) U_{m} U_{i} \frac{d\left(\ln \left(E_{i}^{\prime} \cdot E_{\mathrm{m}}^{\prime}\right)\right)}{d \omega}\right|_{\omega=\omega_{g, m}}
\end{aligned}
$$

Evidently the term $\left.\frac{d\left(\ln \left(E_{i}^{\prime} \cdot E_{\mathrm{m}}^{\prime}\right)\right)}{d \omega}\right|_{\omega=\omega_{g, m}}$ is strictly positive.

Besides, since $E_{i}^{\prime}>E_{\mathrm{m}}^{\prime-1}$ it implies that

$$
\ln \left(E_{i}^{\prime} \cdot E_{\mathrm{m}}^{\prime}\right)=\ln \left(\frac{E_{i}^{\prime}}{E_{\mathrm{m}}^{\prime-1}}\right)>\frac{E_{i}^{\prime}-E_{\mathrm{m}}^{\prime-1}}{E_{i}^{\prime}}
$$

and therefore since the second derivative of the continuous function $\ln (x)$ is strictly negative it follows

$$
\left.\frac{d}{d \omega}\left[\ln \left(E_{i}^{\prime} \cdot E_{\mathrm{m}}^{\prime}\right)\right]\right|_{\omega=\omega_{g, m}}<\frac{d}{d \omega}\left(\frac{E_{i}^{\prime}-E_{\mathrm{m}}^{\prime-1}}{E_{i}^{\prime}}\right)=\left.\frac{d}{d \omega}\left(\frac{E_{\mathrm{m}}^{\prime} E_{i}^{\prime}-1}{E_{\mathrm{m}}^{\prime 2} E_{i}^{\prime}}\right)\right|_{\omega=\omega_{g, m}}
$$

Here, we should emphasize that the above inequality concerns only the algebraic rates of all the involved quantities.

Nevertheless, the term $H(\omega)$ has a negative contribution in eqn. (30) since it has always a positive sign and it is abstracted from the right member of eqn. (30).

Consequently, after introducing these considerations one may conclude that the sign of the term $\left.\frac{d \eta_{L}(\omega)}{d \omega}\right|_{\omega=\omega_{g, m}}$ depends mainly on $\left.\frac{d E_{\mathrm{m}}^{\prime}}{d \omega}\right|_{\omega=\omega_{g, m}} ;\left.\frac{d \eta_{i}}{d \omega}\right|_{\omega=\omega_{g, m}}$ with $\left.\frac{d E_{\mathrm{m}}^{\prime}}{d \omega}\right|_{\omega=\omega_{g, m}}>0$.

Besides, the values $\left.\frac{d \eta_{i}}{d \omega}\right|_{\omega=\omega_{g, m}}$ are interconnected with the relative position of interphase glass transition temperature.

In particular at $T_{g, i}>T_{g, m}$ it implies that $\omega_{g, i}>\omega_{g, m}$ The physical meaning of the latter inequality is that the loss factor of interphase has reached its peak value at a frequency less than $\omega_{g, m}$ and therefore it has already started to diminish.

Thus in such materials where strong bonds occur between filler and polymeric matrix, the interlayer bounds should have glass - transition temperatures larger than the rest of the matrix, although the intermediate phase is somewhat an altered matrix. 


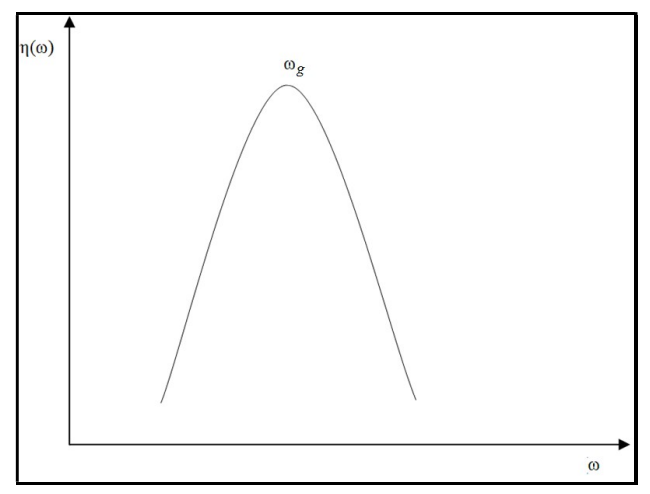

Fig. 6 Qualitative illustration of loss factor vs frequency

\section{Conclusions}

During the estimation of thermomechanical properties of unidirectional fibrous composites by means of various theoretical models, the surface of the fibers is considered as a perfect mathematical one. However, this assumption cannot alleviate the fact that the transition of their values from matrix to fiber is carried out by "jumps" in their characteristic properties. Evidently, this situation is rather unrealistic. Hence, in this study, a model was presented according to which a third phase, the interphase, was taken into account as developed between the two initial phases, during the polymerization process of the matrix. The properties of this intermediate phase depend on those of the primal constituents of the overall material as well as the adhesion quality.

An application of the correspondence principle to this model, led to theoretical expressions for dynamic moduli, in terms of frequency. Then, the loss factor of the overall material was evaluated and examined in the glassy region. The total value of the composite $T_{g, L}$ is an upper limit for this structurally important region and was examined as combination of $T_{g, i} ; T_{g, m}$. Thus, when $T_{g, i}>T_{g, m}$ the derivative of $T_{g, L}$ for the overall material with respect to frequency is positive which implies that the glass transition temperature of the composite increases with frequency. The opposite occurs when $T_{g, i}<T_{g, m}$.

This phenomenon, depending on the mechanical imperfections between matrix and fibers, presents different degrees of strength and thus may counterbalance merely or totally the reinforcing effect of the dispersed fibers of the overall material.

\section{References}

1. T.W. Clyne, P.J. Withers, “An Introduction to composites”, Cambridge

University Press, Cambridge, (1993).

2. Theocaris P.S. The mesophase concept in composites, in Advanced Polymer Science, edited by Kausch H. H. \& Zachmann H. G., (Springer-Verlag), 66 (1985) 149.

3. Theocaris, P. S.: Concept of Mesophase, Mechanisms of Crack Propagation, in: Metal Filled Polymers: Properties and Applications, Battacharya, S. K. (Ed.), Dekker, N. Y., Chap. 5, pp. 259-333 (1986)

4. Papanicolaou,G.C., Theocaris,P.S., Spathis, G.D.: Adhesion Efficiency Between Phases in FiberReinforced Polymers by Means of the Concept of Boundary Interphase, Col. and Polym. Sci. 258, pp. 1231-1237, (1980) 
5. Kanaun, S.K. and Kudriavtseva, L.T. Elastic and thermoelastic characteristics of composites reinforced with unidirectional fibre layers. Appl. Math. Mech., 53, 628-636 (1986).

6. Garboczi, E. J. and Bentz D. P. (1997) Analytical formulas for interfacial transition zone properties, Advanced Cement - Based Materials 6, 99-108.

7. Lutz, M.P. and Zimmerman, R.W. (1996) Effect of the interphase zone on the bulk modulus of a particulate composite, Journal of Applied Mechanics, 63, 855-861.

8. Shen, L and Li, J. (2003) Effective elastic moduli of composites reinforced by particle or fiber with an inhomogeneous interface, International Journal of Solids and Structures, 40, 1393-1409.

9. S. Y. Fu, G. Xu, and Y.W. Mai, Compos. Part B 3, 291, 2002

10. Paipetis, S. A., P. S. Theocaris, A. Marchese, Colloid and Polymer Sci. 257, p. 478 (1979).

11. Landel, R. F., T. L. Smith, American Rocket Society Journal, p. 599 (1961).

12. Schwarzl, F.R., On the Mechanical Properties of Unfilled and Filled Elastomers, Central Laboratory, T.N.O. (Delft, Holland).

13. Turner, S., Appl. Mat. Res. p. 10 (1965).

14. Kumins, C. A., J. Roteman, J. Pol. Sci. 1-A, p. 527 (1963).

15. P. S. Theocaris and G. C Papanicolaou Variation of glass transition temperature with direction in unidirectional glass fiber-reinforced compositesColloid \& Polymer Sci. 258, 1044-1051 (1980)

16. T.H. Lee, Y.C.F. Boy, N.L. Loh, Characterization of a fibre reinforced PPS composite by dynamic mechanical analysis: effect of aspect ratio and static stress, Composites Science and Technology 49 (1993) 217-223.

17. J.F. Gerard, S.J. Andrews, C.W. Macosko, Dynamic mechanical measurements: comparison between bending and torsion methods on a graphite-reinforced and a rubber-modified epoxy, Polymer Composites 11 (2) (1990) 90-96.

18. A. Vasquez, M. Ambrustolo, S.M. Moschiar, M.M. Reboredo, J.F. Gerard, Interphase modification in unidirectional glass-fiber epoxy composites, Composites Science and Technology 5 (1998) 549-558.

19. A.R. Sanadi, R.V. Subramanian, V.S. Manoranjan, The interphasial regions in interlayer fiber composites, Polymer Composites 12 (6) (1991) 377-383

20. V.A. Alvarez, M.E. Valdez, A. Vasquez Polymer Testing 22 (2003) 611-615Dynamic mechanical properties and interphase fiber/matrix evaluation of unidirectional glass fiber/epoxy composites

21. N. Saba, M. Jawaid, Othman Y., Alothman B., M. Paridah

A review on dynamic mechanical properties of natural fibre reinforced

polymer composites, Construction and Building Materials 106 (2016) 149-159

22. E.P. Sideridis, J. Venetis Storage and loss moduli of CSM composites at the glass transition region as defined by the concept of interphase, Composites: Mechanics, Computations, Applications: An International Journal, DOI: 10.1615/CompMechComputApplIntJ.2018018555 
23. Hashin Z. Complex moduli of viscoelastic composites-II. Fiber reinforced materials International Journal of Solids and Structures

Volume 6, Issue 6, June 1970, Pages 797-807

24. Theocaris, P. S. The Mesophase Concept in Composites, Polymers/Properties and Applications 11, Springer-Verlag (1987).

25. Lipatov, Y. S.: Physical Chemistry) of Filled Polymers, published by Khimiya (Moscow 1977). Translated from the Russian by R. J. Moseley, International Polymer Science and Technology, Monograph No.2; see also Lipatov, Y. S., Adv. Polym. Sci. 22, pp. I-59, (1977) 